\title{
Focus on focus: lack of coherence between systemic and microvascular indices of oedema formation
}

\author{
Birkitt L. ten Tusscher ${ }^{1}$, Corien Gudden ${ }^{1}$, Suus van Vliet ${ }^{1}$, Bob Smit ${ }^{1}$, Can Ince ${ }^{2,3}$, \\ E. Christiaan Boerma ${ }^{4}$, Harm-Jan S. de Grooth ${ }^{1}$, Paul W.G. Elbers ${ }^{1}$
}

\begin{abstract}
${ }^{1}$ Department of Intensive Care Medicine, Research VUmc Intensive Care (REVIVE), Amsterdam Cardiovascular Sciences (ACS), Amsterdam Infection and Immunity Institute (AI\&II), VU University Medical Center Amsterdam, The Netherlands

${ }^{2}$ Department of Translational Physiology, Academic Medical Center, Amsterdam, The Netherlands ${ }^{3}$ Department of Intensive Care Medicine, Erasmus Medical Center, Rotterdam, The Netherlands ${ }^{4}$ Department of Intensive Care Medicine, Medical Center Leeuwarden, Leeuwarden, The Netherlands
\end{abstract}

\begin{abstract}
Background: Fluid therapy remains a cornerstone of therapy in shock states. However, fluid overloading ultimately results in oedema formation which is related to excess morbidity and mortality. Handheld microscopes are now frequently used to study the sublingual microcirculation. As a corollary, these devices measure focal distance, or surface to capillary distance. Physiologically, this could represent a microvascular index of oedema formation and could have the potential to guide fluid therapy. This potential tool should be investigated, especially given the frequently reported lack of coherence between systemic and microvascular parameters in the critically ill. Therefore, we set out to assess the correlation between microvascular focal distance and systemic indices of oedema formation, specifically fluid balance and weight gain.

Methods: Following ex vivo testing of focal distance measurement reliability, we conducted a prospective observational cohort study in patients admitted to the intensive care unit of our university teaching hospital. We determined surface to capillary distance using sidestream dark field (SDF) and incident dark field (IDF) imaging by assessing the focal distance point or object distance range at which a sharp recording could be made. Measurements were performed in post-cardiac surgery patients and in patients following emergency admission at two time points separated by at least several hours. Data on fluid balance, weight and weight gain were collected simultaneously.

Results: Sixty patients were included. The focal setting, focus point for SDF and the object distance range for IDF did not differ significantly between time points. Focus was not correlated with difference in fluid balance or weight gain. Conclusions: There is a lack of coherence between surface to capillary distance as determined by SDF or IDF imaging and fluid balance or weight gain. Thus, focal distance as a microvascular index of oedema formation cannot currently be used as a proxy for systemic indices of oedema formation. However, given the lack of coherence, further research should determine whether focal distance may provide better guidance for fluid therapy than traditional markers of overzealous fluid administration.
\end{abstract}

Anaesthesiology Intensive Therapy 2017, vol. 49, no 5, 350-357

Key words: oedema, fluid balance, microcirculation, focal setting, SDF imaging, IDF imaging

Fluid resuscitation is a double-edged sword. The association between the amount of fluids administered and complications or outcome may be depicted as a U-shaped curve (Fig. 1). Perioperative medicine was first in recognizing this phenomenon. While goal directed stroke volume optimization using fluids and inotropes was shown to improve outcome, larger amounts of fluids were actually associated with worse outcome as was repeatedly found in the setting 


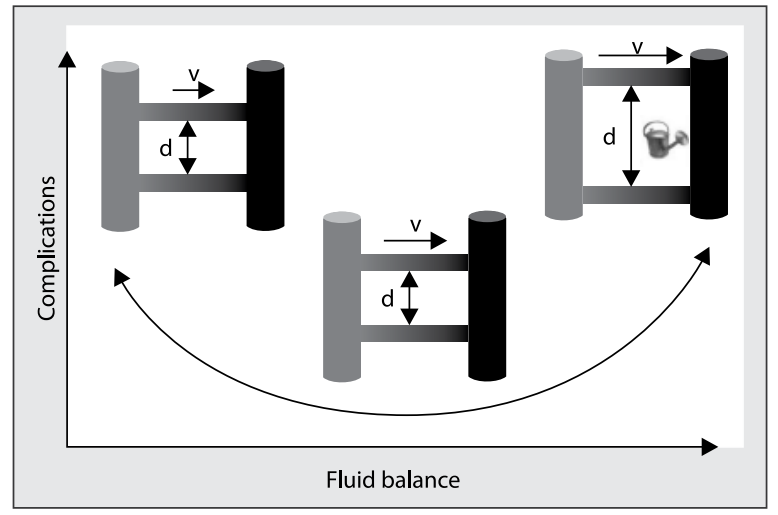

Figure 1. A possible explanation for the relationship between fluid status and complications based from the perspective of microvascular perfusion and the influence of edema formation $\mathrm{V}$ - velocity, $\mathrm{d}$ - diffusion distance. Both too much and too little fluids may lead to decreased oxygen and nutrient delivery to tissue because of poor perfusion and increased diffusion distance caused by edema formation respectively. See text for details

of major surgery [1-3]. In recent years, the possible disadvantages of overzealous fluid administration have also been highlighted in the context of intensive care medicine. In that setting, a positive fluid balance was shown to be associated with impaired organ function and excess mortality [4-7].

The physiological principles underlying the U-shaped curve could be related to fluid induced changes in the microcirculation (Fig. 1). This may be understood by remembering that oxygen transport to tissue is both dependent on convection and diffusion. In that case of absolute or relative volume depletion, cardiac output and microvascular flow velocity are reduced, causing convection to be the limiting factor for oxygen transport. This explains why fluid therapy remains a cornerstone of resuscitation in shock states. However fluid administration will ultimately result in edema. This is thought to increase the distance between capillaries and cells. In that case, diffusion would be the limiting factor for oxygen transport and could explain the negative outcomes associated with positive fluid balance.

In recent years, the development of new generation devices has made real time video monitoring of the human microcirculation feasible. These handheld microscopes are applied sublingually to yield crisp images of red blood cells moving through capillaries (Fig. 2). An easily overlooked feature of these devices is their ability to adjust focal distance by moving the lens (Fig. 2) in order to obtain sharp recordings. As a corollary, this provides a measure of surface to capillary distance ( $\mathrm{SCD}$ ). From the perspective of physiology, SCD can be regarded as a microvascular index of edema. Thus, it could be a clinically relevant marker to guide fluid therapy, especially for deciding when to stop fluid administration.

The assessment of SCD could be a proxy for traditional indices of oedema formation such as fluid balance. If true, this could circumvent the clinical problem of keeping adequate fluid balance which is well known to be cumbersome and error-prone in daily clinical practice of intensive care medicine. In addition, a patient may gain up to $10 \%$ body weight before pitting oedema becomes detectable $[7,8]$.

On the other hand, a consistent finding from microcirculation studies is the frequent lack of coherence between systemic and microvascular parameters. This has strengthened the call for microcirculation guided shock resuscitation [9]. Interestingly, a similar lack of coherence may exist between SCD and systemic indices of oedema formation. This would be of even greater importance as it would lead the way for determining whether SCD is more useful for assessing deleterious effects of overzealous fluid resuscitation than systemic indices. Given the fact that microvascular perfusion is usually assessed sublingually but a good, albeit debated, correlation between sublingual and gut microvascular has been reported, SCD might indeed qualify as a better guide.

Given these considerations we set out to assess the relationship between SCD and fluid balance and weight gain using two popular devices for microvascular imaging (sidestream dark field (SDF) imaging and incident dark field (IDF) Imaging. We hypothesized that this relationship is influenced by severity of illness. Therefore, we included both patients following elective cardiac surgery and following acute admission to intensive care.

\section{METHODS}

We conducted a prospective observational study in the Intensive Care Unit (ICU) of VU University Medical Centre Amsterdam, a university hospital and tertiary referral centre in the Netherlands. The study was approved by the local ethics committee and registered in the Dutch registry for human bound research (CCMO) under trial number NL47067.029.13. Informed consent was obtained from the patient's legal representative and/or if possible from the patients themselves. Enrolment occurred during two periods; from January 2014 to April 2014 and from August 2014 to September 2014 in which SDF and IDF imaging was performed respectively.

\section{PATIENT SELECTION}

Those deemed eligible for inclusion were patients $\geq 18$ years old, admitted to the ICU after elective cardiac surgery (group 1) or following emergency (unplanned) admission (group 2). Exclusion criteria were an inability to access the mouth (e.g. presence of non-invasive ventilation mask), the presence of intra-oral bleeding, evident internal or external bleeding, an incomplete fluid registration or other influences that would have been likely to severely reduce the accuracy of the fluid balance. 


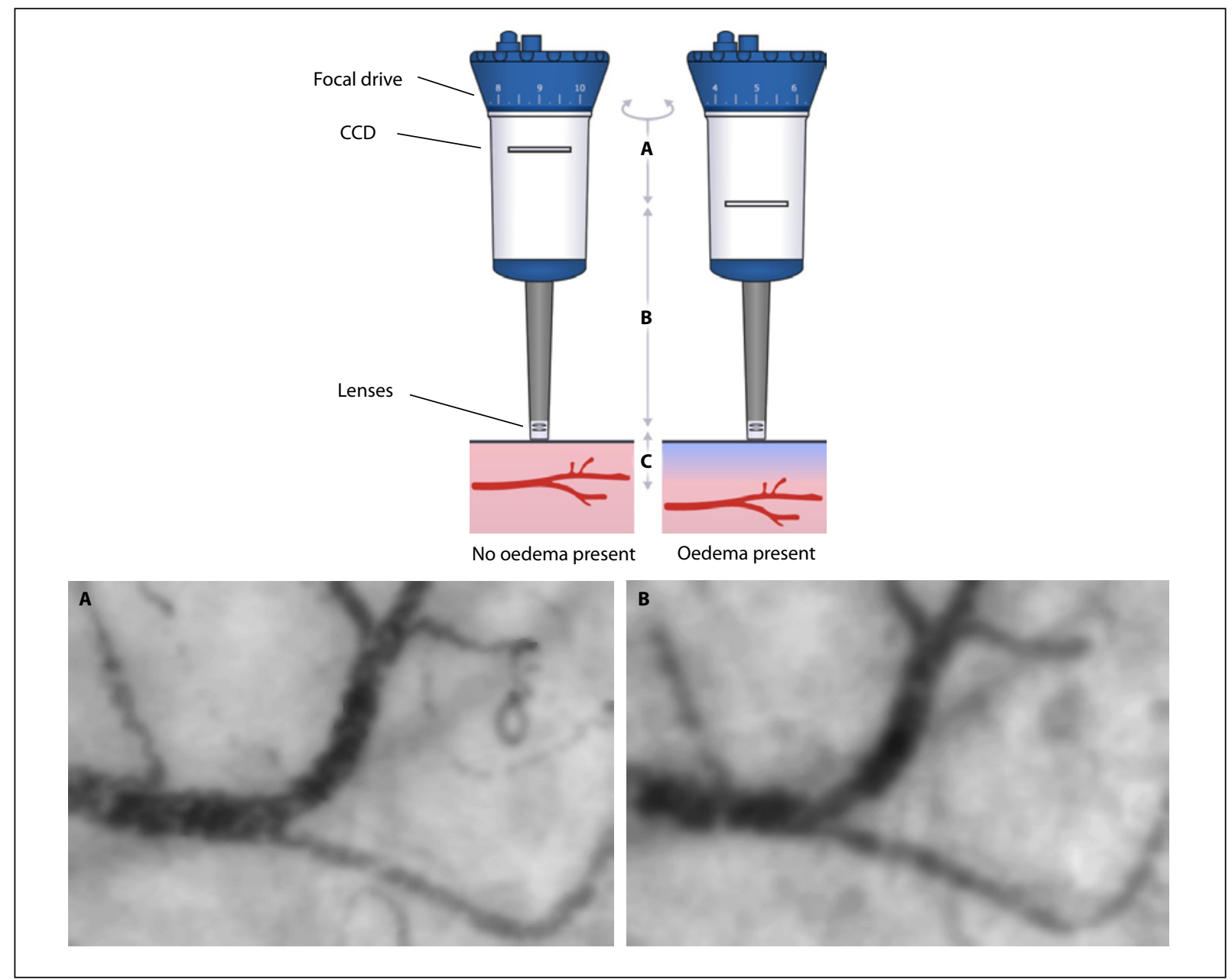

Figure 2. Microvascular imaging using SCD to assess oedema formation using microvascular focal distance. Upper image: oedema may affect the distance between the lens of a microvascular imaging device and the capillaries (object distance, C). Lower image: examples of a sharp image (A) and an image that is out of focus (B)

\section{SCD ASSESSMENT}

Sublingual microcirculatory measurements were performed in two separate periods of inclusion. During each period a single investigator (SV, first period or CG, second period) was responsible for the measurements. During the first period of enrolment sublingual imaging was performed using SDF imaging. A second period of enrolment was added using Incident Dark Field imaging (CytoCam, Braedius Medical B.V., Huizen, the Netherlands), as this device provides factory calibrated servo controlled focal distance reporting.

SDF imaging is based on light- emitting diodes, using a wavelength of $530 \mathrm{~nm}$, the isosbestic point of haemoglobin. This causes both oxy- and deoxyhaemoglobin to fully absorb the light. The result is moving video images of erythrocytes as dark spots on a white/greyish background [10]. During SDF imaging, focus point setting is manually adjusted according to a scale from zero to ten, resulting in the ability to grossly adjust focus point per half point focus distance. The CytoCam device is a new-generation Incident Dark Field (IDF) microscope based on a similar principle as
SDF imaging. However, the image acquisition and sensor are computer-controlled and electronically synchronized to the very short illumination pulses. In addition, IDF imaging uses an additional set of high resolution lenses and a manually controlled motorized focusing system [11]. A recent study demonstrated that Cytocam-IDF provided better image quality than SDF imaging [12] with an extra advantage of allowing its user to directly adjust object distance in order to improve sharpness of the image using steps of $4 \mu \mathrm{m}$. With the IDF microscope a sharp recording can be made over a certain object distance range, instead of the focus point generated with the SDF microscope.

We considered an image optimally sharp when separate erythrocytes could be seen or when transition from vessel edge to tissue was as optimally defined as possible (Fig. 2) $[9,13]$. Microcirculatory imaging was performed after having removed oral secretions using a wet gaze and by preventing pressure artefacts by pulling back the microscope until contact was lost, followed by repositioning of the probe until contact and view was regained [14, 15]. For determination 
of SCD, at each time point 3 measurements were performed at different sublingual sites and the focus point (for SDF microscope) or the object distance range (for the IDF microscope) in which a sharp recording could be made was noted. Although microcirculation itself was not assessed, the image was considered suitable only in the absence of compression artefacts in large microvessels and the absence of thin layers of saliva.

\section{BENCH STUDY}

As only the IDF device provides factory validated precise object distance measurements, we first confirmed that the scale for focal setting to acquire a sharp image with the SDF device is linearly related to the distance between the object and the lens. We took a generic light microscope (Zeiss KF2, Carl Zeiss B.V., Sliedrecht, the Netherlands) with a micrometer fine-drive, removed the binocular tube and objectives and replaced it for the Sidestream Dark Field imaging (SDF, Microvision Medical B.V., Amsterdam, the Netherlands). An object emerged in $0.9 \% \mathrm{NaCl}$ was placed under the SDF device and stepwise the focal setting was adjusted and the relative change in distance between object and lens was registered (Fig. 3). Indeed, object distance had a linear relationship with focal-setting (Pearson's $r=0.99, P<0.001$ ). As the focal-setting was decreased the object distance had to be increased to regain proper focus by an average of 46 $\mu \mathrm{m}$ (SD $10 \mu \mathrm{m})$.

\section{MEASUREMENTS}

We simultaneously recorded focal point setting, fluid balance, SOFA-score, Body Mass Index (BMI), serum albumin and Total Body Water (TBW). The latter was determined using bio impedance analysis (BIA 101 anniversary ASE, AkernSrl, Pontassieve, Italy). To calculate fluid balance in the elective surgery group, the amount of blood loss during surgery was estimated by multiplying the return of the intraoperative blood salvage (IBS) device by 4.0, as suggested by Drummond and Petrovitch [16]. To correct for different body compositions, we calculated the fluid index, defined as the amount of fluids given divided by Body Surface Area (BSA) calculated using the Mosteller formula [17]. Each patient was also examined for the presence of macroscopic oedema.

During the first period of enrolment we only performed measurements at one time point in the elective surgery group, as we assumed cumulative fluid balance during surgery and ICU admission until measurement to adequately represent the overall fluid status as patients were presumed to have a neutral fluid balance before surgery. However, giving the inaccuracy resulting from manual recording of administered fluids or blood loss during surgery, we decided

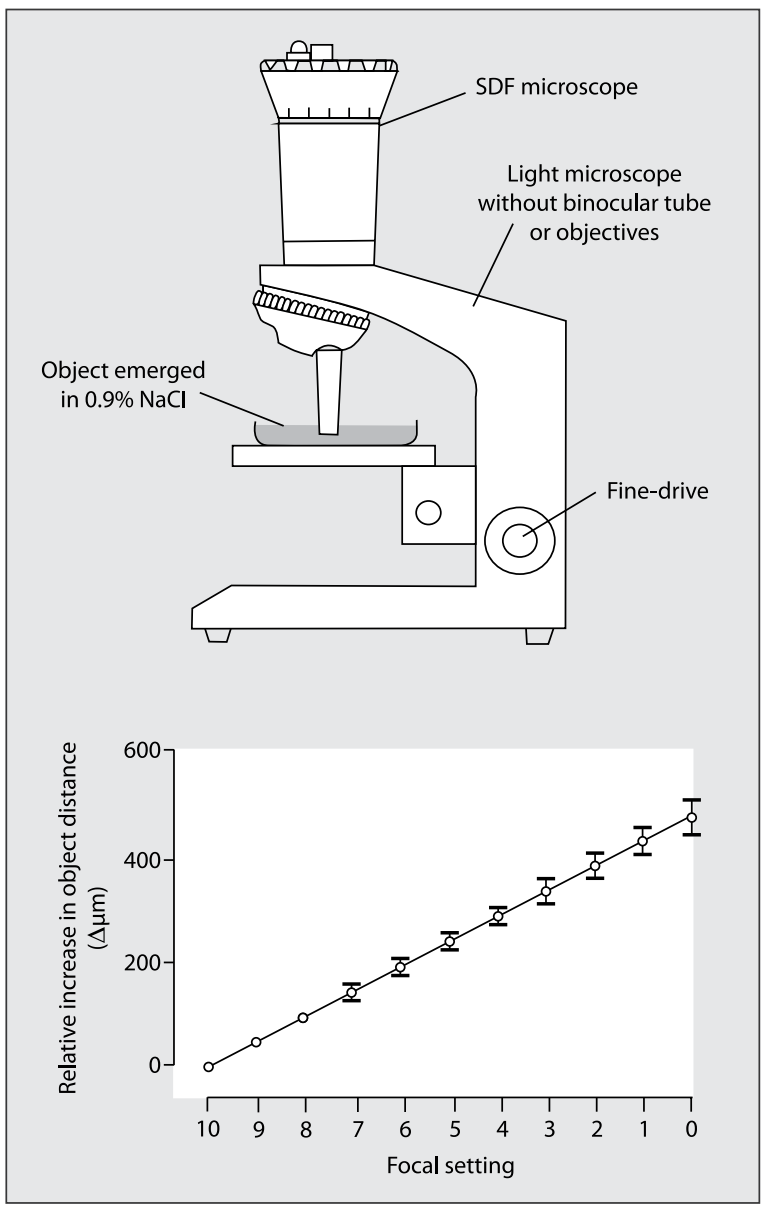

Figure 3. Laboratory setting and effect of focal setting on object to lens distance

to perform measurements at two time points separated by at least several hours in all patients during the second period of enrolment and also included weight gain as a second systemic index of oedema formation.

\section{STATISTICAL ANALYSES AND POWER CALCULATION}

Descriptive statistics were used to compare baseline characteristics in both groups. Normally distributed data were presented as means with standard deviations. Means were compared using Student's t-test. Pearson's correlation was performed to examine associations. Morover $95 \% \mathrm{Cl}$ was calculated using bootstrapping. A $P$-value of $<0.05$ (two-tailed) was considered statistically significant. All statistical analyses were performed using IBM SPSS Statistics version 20.0. We considered a Pearson's $r$ of 0.65 or greater between fluid index and focal point setting to be clinically relevant. With a confidence interval $(\mathrm{Cl})$ of $95 \%$ (alpha 0.05 ) and power of $80 \%$ (beta 0.8 ), this yielded a sample size of 13 patients. To account for missing data, we aimed to include 15 patients per group. 
Table 1. Baseline charasteristics

\begin{tabular}{lcccc}
\hline & SDF elective & SDF urgent & IDF elective & IDF urgent \\
\hline Age [mean (SD)] & $68.4(9.0)$ & $65.3(12.4)$ & $65.1(12.7)$ & $68.6(16.7)$ \\
Male [absolute (percentage)] & $12(80 \%)$ & $9(60 \%)$ & $10(67 \%)$ & $11(73 \%)$ \\
SOFA score (SD) & $4.7(1.3)$ & $8.1(3.3)$ & $5(1.6)$ & $7.5(3.0)$ \\
Mass [kg (SD)] & NA & NA & $81.9(16.1)$ & $93.8(18.0)$ \\
Fluid balance [mL (SD)] & $3293.8(2292.2)$ & $2988.5(2245.9)$ & $1864.9(1589.5)$ & $1945.9(2060.5)$ \\
\hline
\end{tabular}

SD — standard deviation; NA — not available

\section{RESULTS}

\section{SDF IMAGING}

Thirty-four patients were eligible for inclusion. Four patients were excluded; one because of an inability to perform microcirculatory imaging, while one patient withdrew consent during the study and two patients died before second measurements could be performed. Thus, data on fifteen patients after elective surgery and fifteen patients after emergency admission was collected using SDF imaging. Table 1 shows their baseline characteristics

Mean focal setting at the first measurement was 8.5 (range: 6.3-10); 8.2 (range: 6.3-9.5) in elective patients, 8.8 (range: 7.0-10.0) in the emergency admissions group. For the latter, a second measurement was performed, resulting in a mean focal setting of 8.5 (range: 6.7-9.8). Focal setting in the emergency admissions group did not differ between time points (difference $0.35 ; 95 \% \mathrm{Cl}$ : -0.29 to $0.98 ; P=0.261$ ). Moreover, focal setting and difference in fluid balance or corrected fluid balance (Fig. 4) did not show significant correlations. In addition, focal setting was not correlated to the presence of macroscopic oedema, fluid index, BMI, SOFA-score, serum albumin or TBW.

\section{IDF IMAGING}

Thirty-three patients were deemed eligible for inclusion. Three patients were excluded: for one patient, no informed consent could be obtained, while one patient withdrew consent during the study and one patient died before second measurements could be performed. Thus, data on fifteen patients after elective surgery and fifteen patients after emergency admission were collected using IDF imaging. Their baseline characteristics are given in Table 1.

The mean object distance was $119.5 \mu \mathrm{m}$ (range: 74-159 $\mu \mathrm{m}$ ) at the first time point, $123.2 \mu \mathrm{m}$ (range: 80-159 $\mu \mathrm{m}$ ) in the elective surgery group and $115.7 \mu \mathrm{m}$ (range: 74-155 $\mu \mathrm{m})$ in the emergency admissions group. At the second time point, the mean object distance was $127.3 \mu \mathrm{m}$ (range: 90-172 $\mu \mathrm{m}$ ); $127.6 \mu \mathrm{m}$ (range: $95-170 \mu \mathrm{m}$ ) in the elective surgery group and $127.1 \mu \mathrm{m}$ (range: $90-172 \mu \mathrm{m}$ ) in the emergency admissions group.

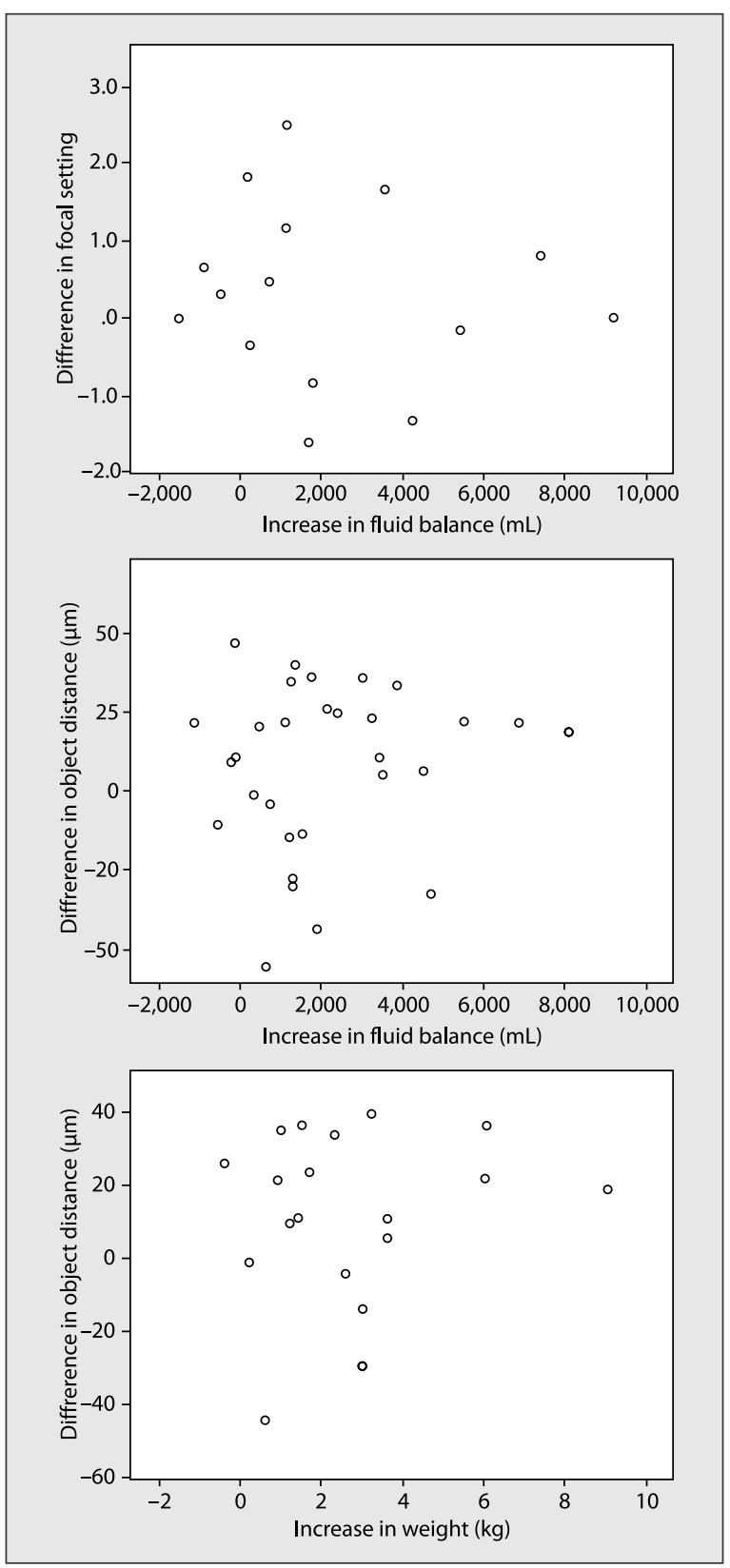

Figure 4. Scatterplots depicting the relationship between the difference in SCD versus increase in fluid balance and weight. Upper plot: difference in focal setting (SDF) vs. increase in fluid balance. Middle plot: difference in object distance (IDF) vs. increase in fluid balance. Lower plot: difference in object distance (IDF) vs. increase in weight 
Object distances in the elective surgery group did not differ between time points (difference: $-4.3 \mu \mathrm{m} ; 95 \% \mathrm{Cl}$ : -19.5 to $10.8 \mu \mathrm{m}, P=0.549$ ), nor did object distances in the emergency admissions group (difference: $-11.4 \mu \mathrm{m}, 95 \% \mathrm{Cl}$ : -25.5 to $2.7 \mu \mathrm{m}, P=0.104)$. Again, there was no correlation between object distance and weight gain or difference in fluid balance or corrected fluid balance (Fig. 4). Moreover, for all time points, object distance was not correlated to the presence of macroscopic oedema, fluid index, BMI, SOFAscore, serum albumin or TBW.

\section{DISCUSSION}

This is the first study to identify the lack of coherence between SCD and fluid balance and weight gain as systemic indices of oedema formation. This was the case for both microvascular imaging devices we used as well as for patients admitted after elective surgery and patients following emergency admission.

From a conceptual point of view, reasons for not finding any associations between microvascular focal distance and systemic indices of oedema formation could include the following: (1) Microvascular imaging devices cannot adequately quantify focal distance and thus interstitial oedema; (2) Clinically relevant oedema formation is not uniformly spread and cannot, therefore, be reliably measured sublingually; (3) SCD may be a clinically more relevant marker for oedema formation that fluid balance or weight gain. The likelihood of the relative contribution of each of these possible explanations is discussed below.

1. An assessment of an optimal focus point arguably remains subjective. The absence of correlation between the focal distance and fluid balance or weight difference, could have been the result of the imprecise adjustment of focal point setting of SDF imaging, which only allows steps of approximately $23 \mu \mathrm{m}$. However, we could also not identify any correlation when using IDF microvascular imaging, which allows more precise adjustment in manufacturer-validated steps of $4 \mu \mathrm{m}$.

We assumed correct placement of the microscope in patients on previous determined quality indicators (pulling back the microscope until contact was lost, followed by repositioning of the probe, removing saliva, confirming absence of pressure artefacts by assessing flow in larger vessels). It is however possible that this positioning is too crude and could influence focal setting, obscuring possible correlation. This could explain the perfect correlation in the ex vivo study using a micrometer fine drive and the absence of a correlation in our patient study. To overcome this problem a future study with an image acquisition stabilizer would be interesting.

2. We chose to assess microcirculation sublingually because it is easily accessible and the burden for the patient is negligible. At each time point, we averaged measurements from 3 different sublingual locations to account for possible local heterogeneity of oedema formation. However, the main question is whether sublingual oedema formation is correlated to oedema formation in other areas of the body especially in the intra-abdominal organs causing the intra-abdominal compartment syndrome and organ damage [4].

The sublingual mucosa shares its embryological origin with the splanchnic mucosa and the sublingual microcirculation has been frequently researched and microvascular dysfunction at this site was repetitively shown to correlate with the severity of the illness $[15,18,19]$. However, whether sublingual microcirculation is representative of that of other organs, especially the gut, remains subject of intense debate and studies with negative as well as positive correlations between sublingual and gut microcirculation in sepsis have been published [20-22]. Therefore, accumulation of oedema, especially in the setting of systemic endothelial leakage, may also not be adequately correlated with sublingual oedema [23]. This could partially explain our findings. In addition, critically ill patients admitted to our ICU usually lie in supine position with slight elevation of the head (approximately 20-30 degrees), possibly resulting in redistribution of oedema from the sublingual site towards deeper located tissue due to gravitational force, which leads to inaccurate measurements sublingually. This could also explain the absence of correlation of oedema on physical examination and focal setting sublingually.

3. Inaccuracy concerning our measurements could have influenced our results. Fluid balance and even weight measurements are prone to error. However, both were meticulously recorded throughout our study. Moreover if inaccurate fluid balance could fully explain the absence of correlation, we would have expected a difference in correlation in the surgery group, with a presumed net fluid balance of zero before surgery and continuous registration of fluid balance during surgery, compared to the emergency admission group with possibly suboptimal registration of fluid balance.

However, even if fluid balance registration could be done very accurately and precisely the problem remains that net positive fluid balance and interstitial oedema formation are not one and the same. There is currently no gold standard for oedema evaluation, especially not for abdominal organs. While fluid balance and oedema correlate well on a population level, they may differ largely between patients. It is possible that our patients had different degrees of oedema that were only marginally related to their different fluid balance. Oedema formation will ultimately depend on the difference between microvascular fluid extravasa- 
tion and lymphatic uptake. While a positive fluid balance will ultimately induce edema, different degrees of capillary permeability and the capacity of the unstressed volume in hypovolaemia, are well known to confound this relationship.

Another consideration is the possible accumulation of fluid as pleural effusions or as free fluid in the abdomen. This would lead to less interstitial oedema development as might be expected given a certain net fluid balance. Further, we did not correct for medication use at time of measurement, which could also be of influence.

Although this study could not demonstrate an association between focus distance sublingually and fluid balance or weight it is important to realize this does not exclude the possibility that focal setting is indeed representative of interstitial oedema at the measured site. It is entirely possible that sublingual focal point setting is more relevant than other surrogate markers for interstitial oedema such as fluid balance or weight, especially if accuracy could be further improved e.g. using image acquisition stabilizers.

Of course, this is speculative. On the other hand, given the now well-established lack of coherence between microvascular and systemic indices of perfusion, it would not come as a major surprise. Naturally, it should be proven and ideally also be shown to hold for the relationship between the indices of oedema formation and functional capillary distance.

It would be of major interest to perform an animal study in order asses focal setting sublingually and in the gut simultaneously and to relate these results to differences between the dry and wet weight of organs. Further research in critically ill patients should focus on unveiling any temporal relationship between microvascular focus distance and organ function, especially renal function and the possibility to use this microvascular index of oedema formation in order to guide fluid therapy in the critically ill.

\section{CONCLUSIONS}

There is a lack of coherence between microvascular and systemic indices of oedema formation. This implies that SCD cannot be used as a proxy for fluid balance or weight gain. On the other hand, given this lack of coherence, and given the fact that SCD is measured at a sublingual site known for its phylogenetic relationship to the gut, this also implies that SCD may be a more interesting marker of clinically relevant oedema than the systemic indices. This should pave the road for future studies to assess the relationship between SCD and clinical outcome and complications to further identify its potential role to guide prevention of overzealous fluid resuscitation.

\section{ACKNOWLEDGEMENTS}

1. We would like to thank the intensive care unit staff of the VU University Medical Centre Amsterdam for their support during conduction and execution of this project.
2. Conflict of interest: $\mathrm{Cl}$ has developed SDF imaging and is listed as inventor on related patents commercialized by MicroVisionMedical (MVM) under a license from the Academic Medical Center (AMC). He has been a consultant for MVM in the past, but has not been involved with this company for more than 5 years now, apart from the fact that he still holds shares. Braedius Medical, a company owned by a relative of $\mathrm{Cl}$, has developed and designed a hand-held microscope called CytoCam-IDF imaging. $\mathrm{Cl}$ has no financial relation with Braedius Medical of any sort, i.e., has never owned shares, or received consultancy or speaker fees from Braedius Medical.

3. Source of funding: Institutional funding only. Braedius Medical provided the IDF Cytocam free of charge for use during this study.

\section{References:}

1. Stewart RM, Park PK, Hunt JP, et al. National Institutes of Health/ National Heart, Lung, and Blood Institute Acute Respiratory Distress Syndrome Clinical Trials Network. Less is more: improved outcomes in surgical patients with conservative fluid administration and central venous catheter monitoring. J Am Coll Surg. 2009; 208(5): 725-735, doi: 10.1016/j.jamcollsurg.2009.01.026, indexed in Pubmed: 19476825.

2. Brandstrup B, Tønnesen H, Beier-Holgersen R, et al. Danish Study Group on Perioperative Fluid Therapy. Effects of intravenous fluid restriction on postoperative complications: comparison of two perioperative fluid regimens: a randomized assessor-blinded multicenter trial. Ann Surg. 2003; 238(5):641-648, doi: 10.1097/01.sla.0000094387.50865.23, indexed in Pubmed: 14578723.

3. de Aguilar-Nascimento JE, Diniz BN, do Carmo AV, et al.Clinical benefits after the implementation of a protocol of restricted perioperative intravenous crystalloid fluids in major abdominal operations. World J Surg. 2009; 33(5): 925-930, doi: 10.1007/s00268-009-9944-2, indexed in Pubmed: 19234737.

4. Cordemans C, De Laet I, Van Regenmortel N, et al. Fluid management in critically ill patients: the role of extravascular lung water, abdominal hypertension, capillary leak, and fluid balance. Ann Intensive Care. 2012; 2(Suppl 1 Diagnosis and management of intra-abdominal hyperten): S1, doi: 10.1186/2110-5820-2-S1-S1, indexed in Pubmed: 22873410.

5. Wiedemann HP, Wheeler AP, Bernard GR, et al. National Heart, Lung, and Blood Institute Acute Respiratory Distress Syndrome (ARDS) Clinical Trials Network. Comparison of two fluid-management strategies in acute lung injury. N Engl J Med. 2006; 354(24): 2564-2575, doi: 10.1056/ NEJMoa062200, indexed in Pubmed: 16714767.

6. Barmparas G, Liou D, Lee D, et al. Impact of positive fluid balance on critically ill surgical patients: a prospective observational study. J Crit Care. 2014; 29(6): 936-941, doi: 10.1016/j.jcrc.2014.06.023, indexed in Pubmed: 25085510.

7. Payen D, de Pont AC, Sakr Y, et al. Sepsis Occurrence in Acutely III Patients (SOAP) Investigators. A positive fluid balance is associated with a worse outcome in patients with acute renal failure. Crit Care. 2008; 12(3): R74, doi: 10.1186/cc6916, indexed in Pubmed: 18533029.

8. Cho S, Atwood JE. Peripheral oedema. Am J Med. 2002; 113(1):580-586.

9. Ince C. The rationale for microcirculatory guided fluid therapy. Curr Opin Crit Care. 2014; 20(3): 301-308, doi: 10.1097/MCC.0000000000000091, indexed in Pubmed: 24758985.

10. Goedhart PT, Khalilzada M, Bezemer R, et al. Sidestream Dark Field (SDF) imaging: a novel stroboscopic LED ring-based imaging modality for clinical assessment of the microcirculation. Opt Express. 2007; 15(23): 15101-15114, indexed in Pubmed: 19550794.

11. Braedius Medical B.V. (2014) How it works, CytoCam-IDF. http://www braedius.com/magnoliaPublic/braedius/products/cytoCam/how-it-works.html (3.10.2014).

12. Aykut G, Veenstra G, Scorcella C, et al. Cytocam-IDF (incident dark field illumination) imaging for bedside monitoring of the microcirculation. Intensive Care Med Exp. 2015; 3(1): 40, doi: 10.1186/s40635-015-0040-7, indexed in Pubmed: 26215807. 
13. Massey MJ, Larochelle E, Najarro G, et al. The microcirculation image quality score: development and preliminary evaluation of a proposed approach to grading quality of image acquisition for bedside videomicroscopy. J Crit Care. 2013; 28(6): 913-917, doi: 10.1016/j. jcrc.2013.06.015, indexed in Pubmed: 23972316.

14. De Backer D, Hollenberg S, Boerma C, et al. How to evaluate the microcirculation: report of a round table conference. Crit Care. 2007; 11(5): R101, doi: 10.1186/cc6118, indexed in Pubmed: 17845716.

15. Trzeciak S, McCoy JV, Phillip Dellinger R, et al. Microcirculatory Alterations in Resuscitation and Shock (MARS) investigators. Early increases in microcirculatory perfusion during protocol-directed resuscitation are associated with reduced multi-organ failure at $24 \mathrm{~h}$ in patients with sepsis. Intensive Care Med. 2008; 34(12): 2210-2217, doi: 10.1007/ s00134-008-1193-6, indexed in Pubmed: 18594793.

16. Drummond JC, Petrovitch CT. Intraoperative blood salvage: fluid replacement calculations. Anesth Analg. 2005; 100(3): 645-649, doi: 10.1213/01.ANE.0000144069.36647.3D, indexed in Pubmed: 15728044.

17. Mosteller RD. Simplified calculation of body-surface area. N Engl J Med. 1987; 317(17): 1098, doi: 10.1056/NEJM198710223171717, indexed in Pubmed: 3657876.

18. De Backer D, Creteur J, Preiser JC, et al. Microvascular blood flow is altered in patients with sepsis. Am J Respir Crit Care Med. 2002; 166(1): 98-104, indexed in Pubmed: 12091178.

19. Sakr Y, Dubois MJ, De Backer D, et al. Persistent microcirculatory alterations are associated with organ failure and death in patients with septic shock. Crit Care Med. 2004; 32(9): 1825-1831, indexed in Pubmed: 15343008

20. Verdant CL, De Backer D, Bruhn A, et al. Evaluation of sublingual and gut mucosal microcirculation in sepsis: a quantitative analysis. Crit Care
Med. 2009; 37(11):2875-2881, doi: 10.1097/CCM.0b013e3181b029c1 indexed in Pubmed: 19770750.

21. Boerma EC, van der Voort PHJ, Spronk PE, et al. Relationship between sublingual and intestinal microcirculatory perfusion in patients with abdominal sepsis. Crit Care Med. 2007; 35(4): 1055-1060, doi: 10.1097/01. CCM.0000259527.89927.F9, indexed in Pubmed: 17334238.

22. Pranskunas A, Pilvinis V, Dambrauskas Z, et al. Early course of microcirculatory perfusion in eye and digestive tract during hypodynamic sepsis. Crit Care. 2012; 16(3): R83, doi: 10.1186/cc11341, indexed in Pubmed: 22587828.

23. Klijn E, Den Uil CA, Bakker J, et al. The heterogeneity of the microcirculation in critical illness. Clin Chest Med. 2008; 29(4): 643-54, viii, doi: 10.1016/j.ccm.2008.06.008, indexed in Pubmed: 18954699.

\section{Corresponding author:}

Paul W.G. Elbers

Department of Intensive Care Medicine

Research VUmc Intensive Care (REVIVE)

Amsterdam Cardiovascular Sciences (ACS)

Amsterdam Infection and Immunity Institute (AI\&II)

VU University Medical Center Amsterdam

De Boelelaan 1117, 1081 HV Amsterdam, The Netherlands

e-mail:p.elbers@vumc.nl

Received: 14.09.2017

Accepted: 15.11.2017 